\title{
Die am besten Geeigneten selektionieren
}

\section{Anne Baroffio, Margaret Gerbase, Mathieu Nendaz}

Fachbereich Forschung und Entwicklung in der Medizinausbildung (Unité de Développement et de Recherche en Education Médicale [UDREM]), medizinische Fakultät, Universität Genf

\begin{abstract}
Die Selektion der Kandidatinnen und Kandidaten für das Medizinstudium ist ein topaktuelles, komplexes Thema [1]. Wir können uns der Analyse von Dr. Jacques de Haller zu dieser Thematik und zur Art und Weise, wie das entsprechende Prozedere in der Schweiz abläuft, nur anschliessen. Die medizinische Fakultät der Universität Genf kennt keinen Numerus clausus, da das Universitätsgesetz des Kantons den freien Universitätszugang garantiert. Daraus ergibt sich in der Konsequenz, dass die Selektion am Ende des ersten Studienjahres erfolgt und - wie dies Dr. de Haller feststellt - nach Kriterien, die ebenso fragwürdig sind wie diejenigen des in den deutschsprachigen Kantonen üblichen Eignungstests. Letzterer will die Gesamtheit aller in der Schulzeit erworbenen kognitiven Kenntnisse messen, während unsere Prüfung zum Ende des ersten Studienjahres als Multiple Choice angelegt ist und die im Jahresverlauf erworbenen Kenntnisse prüft.
\end{abstract}

\section{Selektionieren wir Merkmale, die wir als «wünschenswert» erachten (oder bestrafen wir sie nicht vielmehr)?}

Korrespondenz: Université de Genève 24, rue du Général-Dufour CH-1211 Genf 4 Tel. 0223797111 Fax 0223791134
Das Auswahlverfahren ist zwar ein wichtiges Thema, und wir werden noch darauf zurückkommen. Als weitere Herausforderung sind jedoch auch die Kompetenzen zu sehen, die sich die Studierenden in den Studiengängen aneignen. In Bezug auf die Selektion der Studierenden kann Genf kaum als Modell bezeichnet werden. Dagegen darf die medizinische Fakultät der Universität Genf aufgrund der 1995 erfolgten Änderung der Studienordnung in der Schweiz eine Vorreiterrolle beanspruchen [2, 3]. Das erste Studienjahr wurde «medikalisiert», vor allem durch die Einführung eines Programms, das den Bezug zum Arztberuf gewährleistet (Person, Gesundheit, Gesellschaft) und das «Ausmisten» einer Reihe nicht unmittelbar fachrelevanter Kenntnisse (z. B. Pflanzenbiologie ...). Die Genfer Reform hat es sich zum Ziel gesetzt, beim Studierenden die für einen effizient, professionell und fürsorglich arbeitenden Arzt unabdingbaren Kompetenzen ab dem zweiten Studienjahr zu fördern. Dabei geht es u. a. um Problemlösungen, um klinische Kompetenzen und realitätsnahe Kommunikation (vor allem in nachgestellten Szenarien mit «Patienten»), die Entwicklung von Professionalität und die Ausrichtung auf die Bedürfnisse der Gemeinschaft. Es bleibt zu hoffen, dass dieser Ausbildungsansatz allfällige, durch die Selektion geschaffene Mängel mindert. Aber welche Sicherheiten haben wir dafür?

Im Rahmen unserer Analyse stellt sich die Frage: Wer sind unsere Studierenden? Welches sind ihre kognitiven, vor allem aber auch ihre nichtkognitiven Fähigkeiten (Persönlichkeit, Empathiefähigkeit, Stressbewältigung, Motivationstyp usw. ...)? Wie wirkt sich unsere Selektion am Ende des ersten Studienjahres auf diese Population aus? Selektionieren wir Merkmale, die wir als «wünschenswert» erachten (oder bestrafen wir sie nicht vielmehr)? Können unsere Studierenden diese «wünschenswerten» Eigenschaften dank unseren Studiengängen modifizieren (entwickeln)? Und in Richtung welcher Art von praktischer Tätigkeit orientieren sie sich? Gibt es eine Verknüpfung zwischen dieser Wahl und den persönlichen Eigenschaften der Kandidaten?

All diese Fragen veranlassten uns dazu, die im Jahr 2011 initiierte Kohortenstudie CAPA (Contexte d'Apprentissage et Parcours Académique) durchzuführen. Sie beobachtet 2 Kohorten Studierende über die Dauer ihres Studiums. Dazu wurde bereits eine ganze Reihe von Daten erfasst [4]. Diese machen deutlich, dass sich die Studierendenpopulation zu Beginn des ersten Studienjahres durch mehrere wichtige Merkmale auszeichnet. Das erste Merkmal erfasst die Persönlichkeitsprägung, die wir «Gewissenhaftigkeit» (im Sinne von «gewissenhaft sein») nennen, verbunden mit einem in die Tiefe gehenden Lernansatz und einer Form der Stressbewältigung, die darin besteht, dass versucht wird, sich auf die zu erfüllende Aufgabe zu konzentrieren. Dieses Merkmal ist bei Studierenden, die das erste Jahr geschafft haben und damit selektioniert sind, am häufigsten zu finden. Das zweite Merkmal verknüpft die «emotionale Instabilität» mit der Stressbewältigung über die Emotion. Es findet sich häufiger bei Studierenden, die es nicht schaffen. Ausserdem scheint es eher ein Merkmal von Frauen als von Männern zu sein. Daraus resultiert, dass nur jede dritte Frau selektioniert wird, während es bei den Männern jeder zweite ist. Handelt es sich hierbei um eine Geschlechterdiskriminierung oder eine Austarierung der Männer/ Frauen-Rate (30/70 im ersten Jahr, 45/55 im zweiten für unseren Jahrgang 2011)? Die Interpretation kann diskutiert werden. Das dritte Merkmal verknüpft 


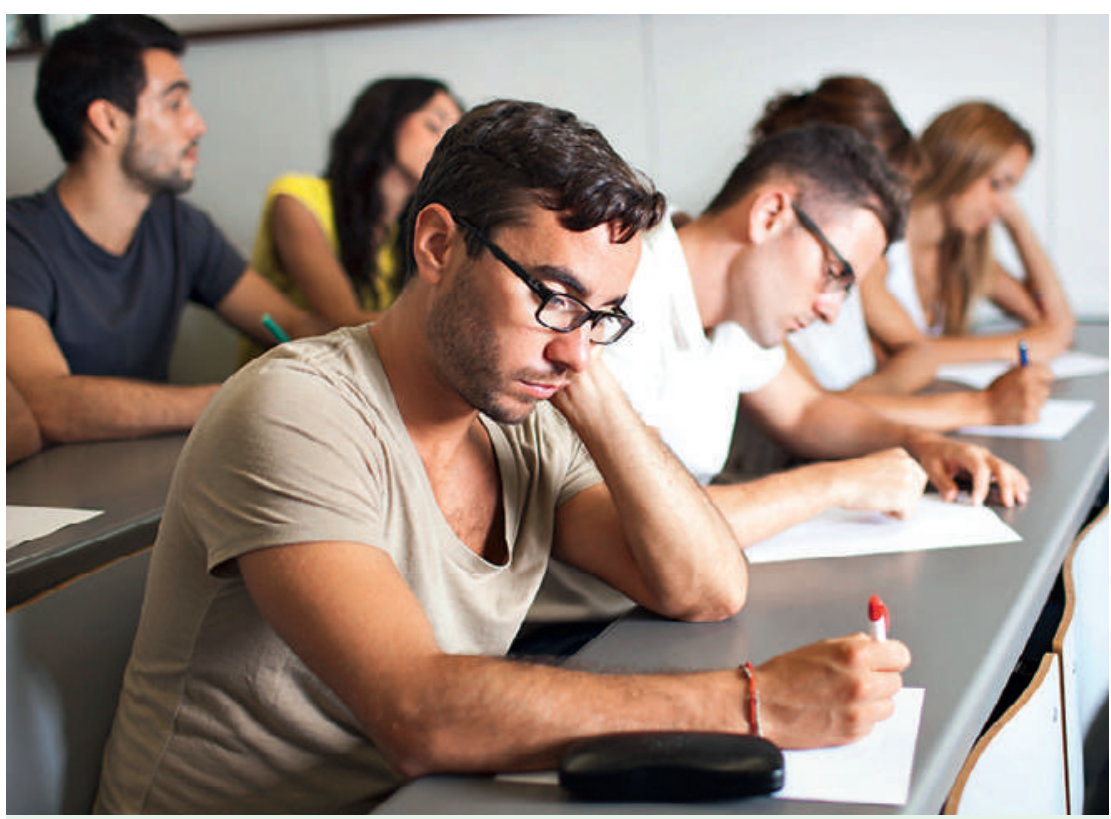

Die medizinische Fakultät der Universität Genf hat keinen Numerus clausus. Die Auswahl der Studierenden erfolgt jeweils am Ende des 1. Studienjahres.

«Umgänglichkeit» und «Extravertiertheit» mit der empathischen Wahrnehmung: Es ist sowohl bei den Selektionierten als auch bei den Nichtselektionierten gleich ausgeprägt. Dies bedeutet, dass es keine Kontraselektion von Eigenschaften gibt, die potentiell für die Beziehungsqualitäten unserer künftigen Ärzte als wichtig erachtet werden.

\section{Multiple Mini-Interviews: ein sehr interessanter, aber relativ teurer Ansatz.}

Diese wenigen, noch vorläufigen Ergebnisse geben lediglich Aufschluss über unsere erste Kohorte von Studierenden während der ersten zwei Studienjahre. In den kommenden fünf Jahren sollen sie ergänzt und vertieft werden. 2013 wurde dieses Projekt vom BAG unterstützt, was das nationale Interesse verdeutlicht, unsere Studierenden besser zu kennen und ihnen die bestmögliche Orientierung zu bieten. Wie Dr. de Haller sind auch wir absolut der Meinung, dass wir nicht darauf verzichten können, unsere Studierenden bereits ab Studienbeginn besser zu selektionieren. Das in Israel angewandte, ursprünglich an der kanadischen MacMaster Universität entwickelte
System der Multiplen Mini-Interviews ist zwar ein sehr interessanter, aber relativ teurer Ansatz. Einen anderen Weg bieten die von Ph. Lievens in Belgien entwickelten Situational Judgment Tests. Bei diesen Tests werden die Kandidatinnen und Kandidaten mit schriftlichen oder gefilmten Szenarien konfrontiert, für die sie anhand einer Liste möglicher Reaktionen eine Lösung wählen müssen. Diese Tests sind deutlich kostengünstiger und haben sich als sehr effizient in der Prüfung der zwischenmenschlichen Beziehungsfähigkeit erwiesen [5]. Noch steht aber der Nachweis, dass sie auch die Selektion der «besten zukünftigen» Ärzte ermöglichen, aus. Vor allem da in einem Alter selektioniert wird, in dem die Persönlichkeit noch stark in der Entwicklung begriffen ist.

Wir müssen also kreativ vorgehen und weiterhin auf Ausbildungsqualität setzen, gleichzeitig aber auch Selektionsmodalitäten umsetzen, die mit der politischen und sozialen Vision der betreffenden Kantone und den Budgets der jeweiligen Universitäten kompatibel sind. Immer mit dem Ziel vor Augen, die geeignetsten Kandidatinnen und Kandidaten zur Entwicklung jener Qualitäten zu selektionieren, die Ärztinnen und Ärzte benötigen, um in einer sich rasch entwickelnden Gesellschaft arbeiten zu können, deren Gesundheitsansprüche sich stetig verändern [6].

Dank: Wir danken Prof. Nu Viet Vu herzlich für ihre Unterstützung und ihr kritisches Lektorat.

\section{Literatur}

1 Mahon KE, Henderson MK, et al. Selecting Tomorrow's Physicians: The Key to the Future Health Care Workforce. Acad Med. 2013;88(12): 1806-11.

$2 \mathrm{Vu}$ N, Bader C, et al. The Redesigned Undergraduate Medical Curriculum at the University of Geneva. Advances in medical education. V. d. V. C. AJJA Scherpbier, Rethans JJ. Dordrecht: Kluwer Academic Publishers; 1997. p. 532-5.

3 Bernheim L, Chastonay P, et al. Les études de Médecine à Genève: Rétrospective et Perspective. Chêne-Bourg: Editions Médecine et Hygiène; 2011.

4 Baroffio A, Abbiati M, et al. Processus de sélection des étudiants en médecine: $y$-a-t-il des profils favorables? Montréal, Québec: Forum de pédagogie des Sciences de la santé; 2013a.

5 Lievens F, Sackett PR (2012). The validity of interpersonal skills assessment via situational judgment tests for predicting academic success and job performance. J Appl Psychol. 2012;97(2):460-8.

6 Cohen JJ. Will changes in the MCAT and USMLE ensure that future physicians have what it takes? JAMA. 2013;310(21):2253-4. 